ARTICLE

https://doi.org/10.1038/s41467-019-12859-2

\title{
A general synthesis approach for amorphous noble metal nanosheets
}

\author{
Geng Wu${ }^{1,9}$, Xusheng Zheng ${ }^{2,9}$, Peixin Cui ${ }^{3,9}$, Hongyu Jiang ${ }^{4}$, Xiaoqian Wang ${ }^{1}$, Yunteng Qu ${ }^{1}$, Wenxing Chen $^{5}$,

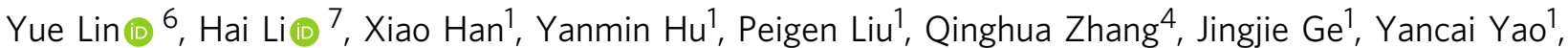 \\ Rongbo Sun', Yuen Wu (i) ${ }^{1}$, Lin Gu (1) ${ }^{4}$, Xun Hong (D) ${ }^{1 \star} \&$ Yadong $\mathrm{Li}^{1,8 \star}$
}

Noble metal nanomaterials have been widely used as catalysts. Common techniques for the synthesis of noble metal often result in crystalline nanostructures. The synthesis of amorphous noble metal nanostructures remains a substantial challenge. We present a general route for preparing dozens of different amorphous noble metal nanosheets with thickness less than $10 \mathrm{~nm}$ by directly annealing the mixture of metal acetylacetonate and alkali salts. Tuning atom arrangement of the noble metals enables to optimize their catalytic properties. Amorphous Ir nanosheets exhibit a superior performance for oxygen evolution reaction under acidic media, achieving 2.5-fold, 17.6-fold improvement in mass activity (at $1.53 \mathrm{~V}$ vs. reversible hydrogen electrode) over crystalline Ir nanosheets and commercial $\mathrm{IrO}_{2}$ catalyst, respectively. In situ X-ray absorption fine structure spectra indicate the valance state of $\mathrm{Ir}$ increased to less than +4 during the oxygen evolution reaction process and recover to its initial state after the reaction.

\footnotetext{
${ }^{1}$ Hefei National Laboratory for Physical Sciences at the Microscale, Department of Applied Chemistry, Center of Advanced Nanocatalysis (CAN), University of Science and Technology of China, 230026 Hefei, Anhui, People's Republic of China. ${ }^{2}$ National Synchrotron Radiation Laboratory (NSRL), University of Science and Technology of China, 230029 Hefei, Anhui, People's Republic of China. ${ }^{3}$ Key Laboratory of Soil Environment and Pollution Remediation, Institute of Soil Science, Chinese Academy of Sciences, 210008 Nanjing, People's Republic of China. ${ }^{4}$ Institute of Physics, Chinese Academy of Sciences, 100190 Beijing, People's Republic of China. ${ }^{5}$ Beijing Key Laboratory of Construction Tailorable Advanced Functional Materials and Green Applications, School of Materials Science and Engineering, Beijing Institute of Technology, 100081 Beijing, People's Republic of China. ${ }^{6}$ Hefei National Laboratory for Physical Sciences at the Microscale, University of Science and Technology of China, 230026 Hefei, People's Republic of China. ${ }^{7}$ Key Laboratory of Flexible Electronics (KLOFE) and Institute of Advanced Materials (IAM), Jiangsu National Synergetic In-novation Center for Advanced Materials (SICAM), Nanjing Technology University, 211816 Nanjing, Jiangsu, People's Republic of China. ${ }^{8}$ Department of Chemistry, Tsinghua University, 100084 Beijing, People's Republic of China.

${ }^{9}$ These authors contributed equally: Geng Wu, Xusheng Zheng, Peixin Cui. *email: hongxun@ustc.edu.cn; ydli@mail.tsinghua.edu.cn
} 
$\mathrm{N}$ oble metal nanomaterials possess potential applications in catalysis $^{1-3}$, energy storage, and conversion ${ }^{4-6}$. For example, Ir and its oxide nanomaterials are unanimously considered as the most promising catalysts for oxygen evolution reaction (OER) under acidic media, which is the primary bottleneck in electrolysis of water due to the intrinsically sluggish kinetics ${ }^{7-9}$. Unfortunately, the scarcity and expensiveness of noble metals greatly hamper their widespread application. Therefore, tremendous efforts have been devoted to constructing more active noble metal catalysts by increasing the number of active sites or optimizing the intrinsic activity ${ }^{7}$. To this end, how the size $^{10}$, shape ${ }^{11,12}$, and crystal phase ${ }^{13-15}$ of noble nanostructures influence their catalytic performance have been well investigated. As the adsorption/desorption kinetics of intermediate species depend well on the surface structure of catalysts $^{16}$, the construction of catalysts surface structure ${ }^{17-19}$, especially on the atomic level, has emerged as an efficient and successful strategy to further enhance the catalytic performance of noble metal nanomaterials.

Distinctive from crystalline materials with translational periodicity, the special disorder atomic structure with the lack of long-range rotational and translational symmetry ${ }^{20,21}$ endows amorphous materials with unique properties ${ }^{22-24}$. For example, amorphous materials possess superior elastic strain performance, which is attributed to the chemical fluctuations and local topological in the amorphous structure ${ }^{22}$. Moreover, the large amount of randomly oriented bonds enable amorphous materials with abundant defects and coordinationunsaturated sites on the surface ${ }^{25-28}$, potentially providing superior catalytic performance than crystalline counterparts. For instance, amorphization can efficiently promote the intrinsic properties of $\mathrm{Pd}_{3} \mathrm{P}_{2} \mathrm{~S}_{8}$ for the electrocatalytic hydrogen evolution reaction and amorphous gelled FeCoW oxyhydroxides exhibit a remarkable performance for OER in alkaline electrolyte compared with crystalline counterparts ${ }^{29,30}$. Unfortunately, since the strong and isotropic nature of metallic bonds ${ }^{31}$, noble metal nanomaterials synthesized by conventional strategies are usually crystalline $\mathrm{e}^{32}$. Therefore, the synthesis of amorphous noble metal nanomaterials where elemental composition, material size, and shape can be precisely controlled remains substantial challenges.

Herein, we demonstrate a general and facile method to synthesize amorphous noble metal nanosheets (NSs) via directly annealing metal acetylacetonates with alkali salt (Fig. 1 and Supplementary Table 1). The synthetic temperature is situated between the melt point of metal acetylacetonate and that of alkali salt. After removing alkali salt with water, high-yield amorphous noble metal-based NSs have been obtained, including but not limited to monometal NSs (Ir NSs, Rh NSs, Ru NSs), bimetal NSs (RhFe NSs, IrRu NSs), and trimetal NSs (IrRhRu NSs). The obtained amorphous Ir NSs achieve exceptional catalytic activity when benchmarked again commercial catalysts for electrochemical OER under acidic media.

\section{Results}

Synthesis and characterization of amorphous Ir NSs. Amorphous Ir NSs have been prepared by annealing the mixture of iridium acetylacetonates $\left(\operatorname{Ir}(\mathrm{acac})_{3}\right)$ and alkali nitrate $\left(\mathrm{KNO}_{3}\right)$ in air. Typical transmission electron microscopy (TEM) image (Fig. 2a) and scanning electron microscopy (SEM) image (Supplementary Fig. 1) reveal the lateral size of obtained NSs controls up to a few micrometers. Atomic force microscopy (AFM) image (Supplementary Fig. 2) displays that the thickness of NSs is $\sim 7.2$ nm. High-angle annular dark-field scanning TEM (HAADFSTEM) image and the corresponding energy-dispersive X-ray spectroscopy (STEM-EDS) elemental mapping (Fig. 2b) confirm that Ir and C are homogeneously distributed within whole NSs. Quantitative EDS spectrum (Supplementary Fig. 3) reveals that the Ir content in NSs is up to $84 \mathrm{wt} \%$. Aberration-corrected HAADF-STEM image (Fig. 2c) shows the disordered atomic structure, verifying the amorphous feature, which accords with the diffractive halo-like selected area electron diffraction (SAED) pattern (the inset in Fig. 2c). These data coincide well with the Xray diffraction (XRD) pattern (Supplementary Fig. 4), in which diffraction peaks for neither Ir nor $\mathrm{IrO}_{2}$ crystal can be detected. As depicted in X-ray photoelectron spectroscopy (XPS) spectra (Supplementary Fig. 5), the peaks located at 61.0 and $63.9 \mathrm{eV}$ are assigned to $\operatorname{Ir}\left(4 f_{7 / 2}\right)$ and $\operatorname{Ir}\left(4 f_{5 / 2}\right)$ of $\operatorname{Ir}^{0}$ and the peaks located at 62.1 and $65.0 \mathrm{eV}$ are assigned to $\operatorname{Ir}\left(4 f_{7 / 2}\right)$ and $\operatorname{Ir}\left(4 f_{5 / 2}\right)$ of $\operatorname{Ir}^{4+}$, which can be contributed to partial oxidation of Ir under air atmosphere, respectively 33,34 . To quantify the local structural characterization of samples, the radial distribution function (RDF) was obtained from SAED patterns of amorphous and crystalline Ir NSs by using the PASAD tools ${ }^{35}$. As shown in the RDF plots (Fig. $2 \mathrm{~d})$, the first two peaks $\left(R_{\text {nea }}\right.$ and $\left.R_{\text {sec }}\right)$ reflect to the average distance of the nearest and the second nearestneighbor Ir atoms, respectively. Notably, in the case of amorphous NSs, the $R_{\text {nea }}$ peak position is shifted to higher distances ( $2.92 \AA)$ compared to the crystalline NSs $(2.68 \AA)$. Furthermore, distinct from those for the crystalline NSs, the $R_{\text {nea }}$ and $R_{\text {sec }}$ peaks in amorphous NSs are broadened and shifted toward higher distances. The observed deviation of RDF peak positions and their significant broadening for amorphous NSs indicate that the corresponding atomic structure has a poor periodicity. Extended $\mathrm{X}$-ray absorption fine structure (EXAFS) spectrum and X-ray absorption near-edge structure (XANES) spectrum of $\mathrm{Ir}_{3}$-edge were employed to further investigate the atomic structure of amorphous NSs (Fig. 2e-g, Supplementary Figs. 6, 7). The peak around $2.92 \AA$ attributed to Ir-Ir bonds in amorphous NSs is slightly larger than that in Ir powder $(2.71 \AA)$, presumably resulting from the loose packing characteristic of non-crystalline state $^{25,36}$. On the basis of the curve-fitting analysis of the EXAFS spectrum (Fig. 2e, g), the coordination number of Ir-C/O and IrIr in the amorphous Ir NSs are 5.1 and 6.3, respectively. Moreover, the wavelet spectrum of amorphous Ir NSs (Fig. 2f) contains two obvious intensity of $\mathrm{Ir}-\mathrm{C} / \mathrm{O}$ and $\mathrm{Ir}-\mathrm{Ir}$ coordination, in line with the corresponding curve-fitting results.

Characterization of other amorphous noble metal NSs. To further expand applicability of the synthetic method, amorphous Rh NSs with a thickness of about $5.3 \mathrm{~nm}$ and amorphous Ru NSs with a thickness of about $5.7 \mathrm{~nm}$ were effectively prepared and corroborated by TEM, SEM, and HAADF-STEM images (Fig. 3a-c, e-g) as well as XRD pattern (Supplementary Fig. 8). Similarly, the homogeneous spatial distributions of metal element and carbon in amorphous noble metal NSs can be also confirmed by EDS elemental mappings (Fig. 3d, h and Supplementary Fig. 9, 10). Besides monometal amorphous NSs, bimetal amorphous NSs can be also fabricated by the similar approach. For example, amorphous RhFe bimetallic NSs with a thickness of about $7.6 \mathrm{~nm}$ and IrRu bimetallic NSs with a thickness of about $11.5 \mathrm{~nm}$ were successfully obtained (Fig. 3i-p and Supplementary Figs. 8, 11, 12). Analogously, a wide variety of bimetal and even trimetal amorphous NSs, such as amorphous $\mathrm{IrFe}$ ( $\mathrm{IrNi}, \mathrm{IrCo}) \mathrm{NSs}, \mathrm{RhNi}$ (RhCo, RhRu) NSs, RuFe (RuNi, RuCo) NSs, IrRh NSs, and IrRhRu NSs, can also be conveniently prepared (Supplementary Figs. 13-15). Furthermore, the thickness control of amorphous NSs could be also achieved, as demonstrated by the synthesis of amorphous Ir NSs with a thickness of $3.6 \mathrm{~nm}$ (Supplementary Figs. 16-18). 


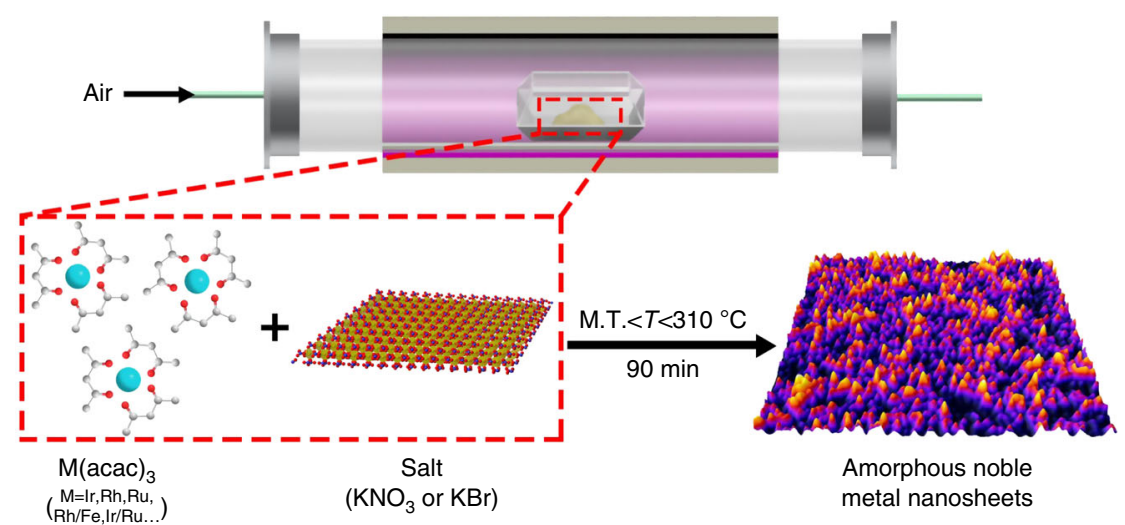

Fig. 1 Schematic illustration of the general synthetic process for amorphous noble metal NSs. Note: M.T. is the melting point of metal acetylacetonate
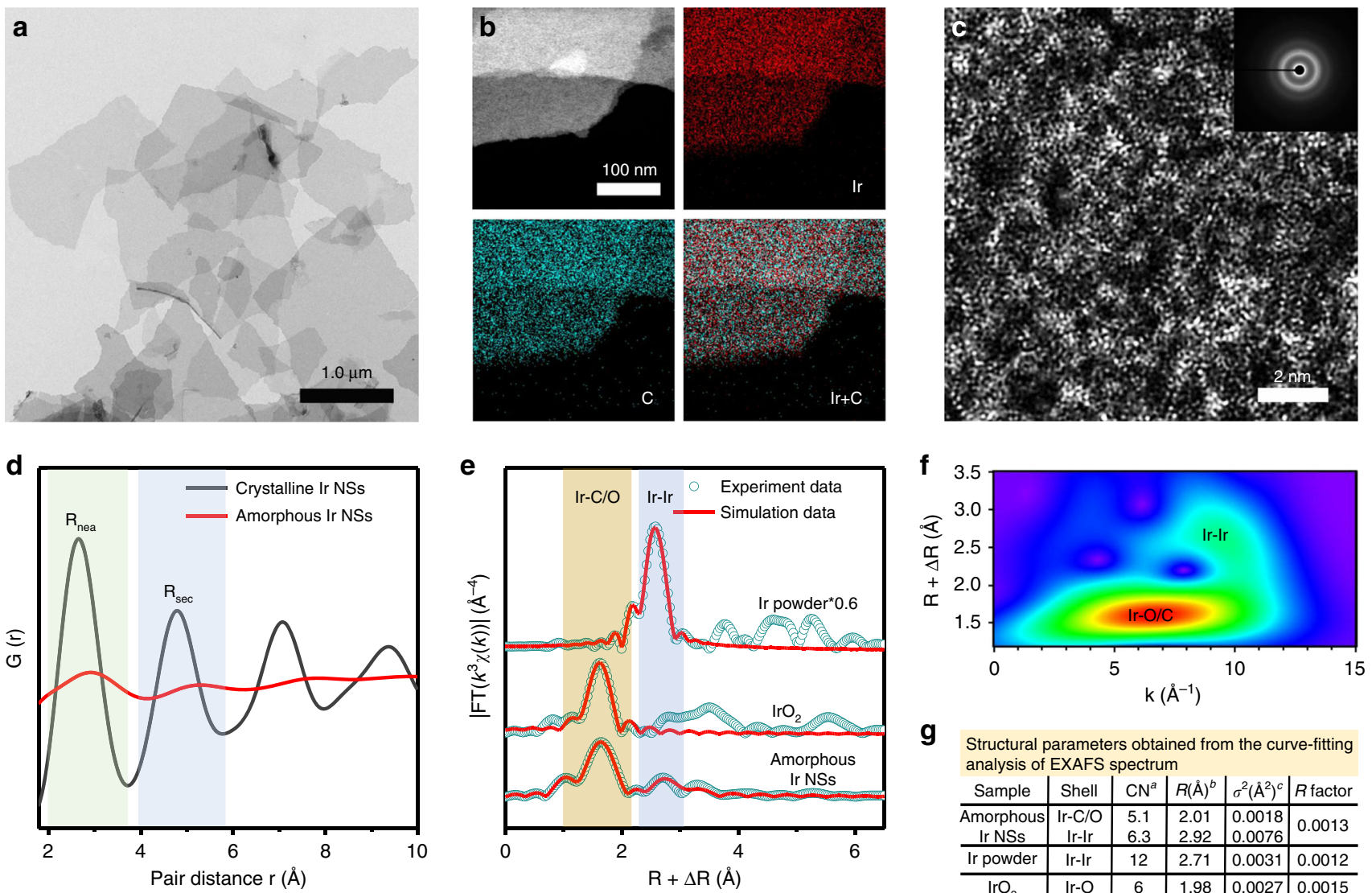

g

Structural parameters obtained from the curve-fitting analysis of EXAFS spectrum

\begin{tabular}{c|c|c|c|c|c} 
Sample & Shell & $\mathrm{CN}^{a}$ & $R\left(\AA \AA^{b}\right)^{b}$ & $\sigma^{2}\left(\AA^{2}\right)^{c}$ & $R$ factor \\
\hline Amorphous & $\mathrm{Ir}-\mathrm{C} / \mathrm{O}$ & 5.1 & 2.01 & 0.0018 & 0.0013 \\
Ir NSs & $\operatorname{Ir}-\mathrm{Ir}$ & 6.3 & 2.92 & 0.0076 & \\
\hline Ir powder & $\mathrm{Ir}-\mathrm{Ir}$ & 12 & 2.71 & 0.0031 & 0.0012 \\
\hline $\mathrm{IrO}_{2}$ & $\mathrm{Ir}-\mathrm{O}$ & 6 & 1.98 & 0.0027 & 0.0015 \\
\hline
\end{tabular}

Fig. 2 Characterizations of amorphous Ir NSs. a TEM, b HAADF-STEM image and the corresponding EDS elemental mapping, c aberration-corrected HAADF-STEM image of amorphous Ir NSs. The inset in c shows the SAED pattern. d Radial distribution functions of amorphous Ir NSs and crystalline Ir NSs. e Fourier transforms of $\mathrm{k}_{3}$-weighted Ir $\mathrm{L}_{3}$-edge EXAFS experimental data for amorphous Ir NSs, Ir powder and Ir $\mathrm{O}_{2}$. $\mathbf{f} \mathrm{Wavelet}$ transform of Ir $\mathrm{L}_{3}$-edge EXAFS data for amorphous Ir NSs. $\mathbf{g}$ Structural parameters obtained from the curve-fitting analysis of the EXAFS spectrum. Note: aCN is the coordination number. ${ }^{b} R$ is interatomic distance (the bond length between central atoms and surrounding coordination atoms). ${ }^{C} \sigma^{2}$ is Debye-Waller factor (a measure of thermal and static disorder in absorber-scatterer distances)

Taking amorphous Ir NSs as a representative example, control experiment with different parameters, including temperature, salt substrate, atmosphere, and annealing time, were further carried out for an in-depth understanding of the growth processes. First, temperature is critical for the formation of amorphous NSs, which must be situated between the melt point of metal acetylacetonate and that of alkali salt. The annealing temperature above the melt point of alkali salt leads to the production of crystalline materials (Supplementary Fig. 19), while an annealing temperature below the melt point of metal acetylacetonate cannot drive the reaction. Second, alkali salt is quite essential for controlling the formation of amorphous NSs. In the absence of alkali salt, crystalline Ir nanoparticles were obtained via directly annealing $\operatorname{Ir}(\mathrm{acac})_{3}$ (Supplementary Fig. 20), indicating that noble metal can be in situ reduced by carbon originated from the thermal decomposition of metal acetylacetonate. When changing 

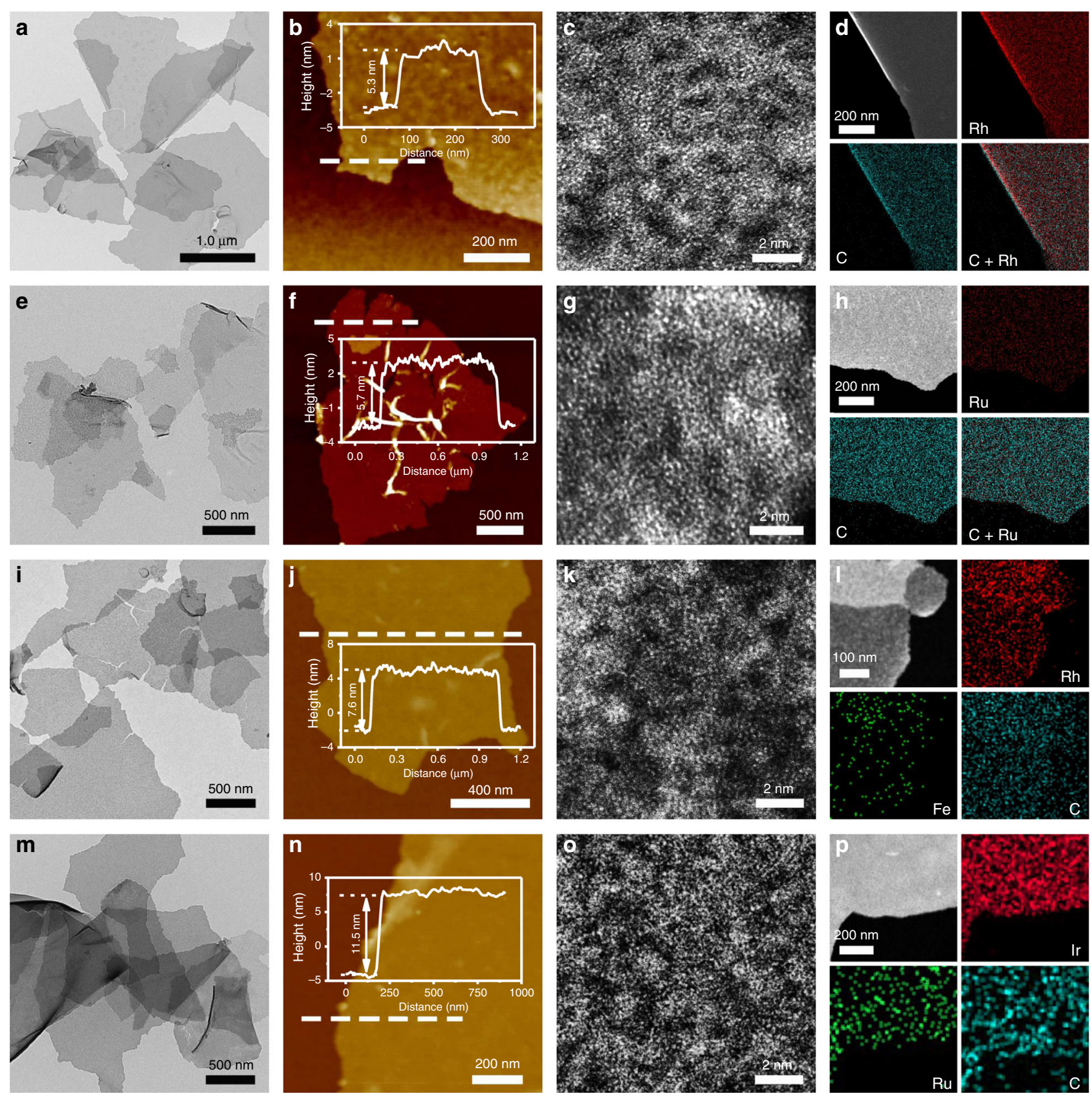

Fig. 3 Characterizations of other amorphous noble metal NSs. $\mathbf{a}, \mathbf{e}, \mathbf{i}, \mathbf{m}$ TEM, $\mathbf{b}, \mathbf{f}, \mathbf{j}, \mathbf{n}$ AFM, $\mathbf{c}, \mathbf{g}, \mathbf{k}, \mathbf{o}$ aberration-corrected HAADF-STEM image, and $\mathbf{d}, \mathbf{h}, \mathbf{I}$, p HAADF-STEM image and the corresponding EDS elemental mapping of amorphous Rh NSs, Ru NSs, RhFe NSs, and IrRu NSs

alkali salt substrate from $\mathrm{KNO}_{3}$ to $\mathrm{NaCl}$ (or $\mathrm{KBr}$ ), crystalline $\mathrm{Ir}$ NSs were synthesized instead of amorphous products (Supplementary Figs. 21, 22). The change of structure might be attributed to the different diffusion rate of Ir atoms on the substrate surface, while the low diffusion rate is beneficial to form amorphous structure during the nucleation process ${ }^{37,38}$. Third, carbonsupported crystalline Ir nanoparticles were prepared by changing the atmosphere from air to argon (Supplementary Fig. 23). Combined by EXAFS spectra and XPS data, the Ir-C/O coordination that existed in amorphous Ir NSs might help in maintaining the amorphous structure. Furthermore, crystalline Ir NSs were formed through extending heat time (Supplementary Fig. 24).

Electrocatalytic activity of amorphous Ir NSs towards OER. As a proof of concept, we chose acidic OER to evaluate the electrocatalytic performance of amorphous Ir NSs. Crystalline Ir NSs (see Supplementary Methods for experimental details and Supplementary Fig. 22), and commercial $\mathrm{RuO}_{2}$ and $\mathrm{IrO}_{2}$ catalysts (Supplementary Fig. 25) are employed as references. As shown in polarization curves normalized by glassy carbon electrode (GCE) geometric area $\left(0.196 \mathrm{~cm}^{2}\right)$ (Fig. $\left.4 \mathrm{a}\right)$, the amorphous Ir NSs require an overpotential of only $255 \mathrm{mV}$ to achieve a current density of $10 \mathrm{~mA} \mathrm{~cm}^{-2}$, which is lower than that of crystalline Ir NSs $(280 \mathrm{mV}), \mathrm{RuO}_{2}(301 \mathrm{mV})$, and $\mathrm{IrO}_{2}(373 \mathrm{mV})$ catalysts. To evaluate the kinetic behaviors of the electrocatalysts, the Tafel plots of elerctrocatalysts (Fig. 4b) exhibit slopes of 40, 55, 73, and $112 \mathrm{mV} \mathrm{dec}^{-1}$ for amorphous Ir NSs, crystalline Ir NSs, $\mathrm{RuO}_{2}$, and $\mathrm{IrO}_{2}$ catalysts, respectively. The considerably smaller slope achieved by the amorphous Ir NSs indicates significantly improved kinetics towards electrochemical OER. Remarkably, amorphous Ir NSs deliver a high mass activity of $221.8 \mathrm{~A} \mathrm{~g}^{-1}$ at 

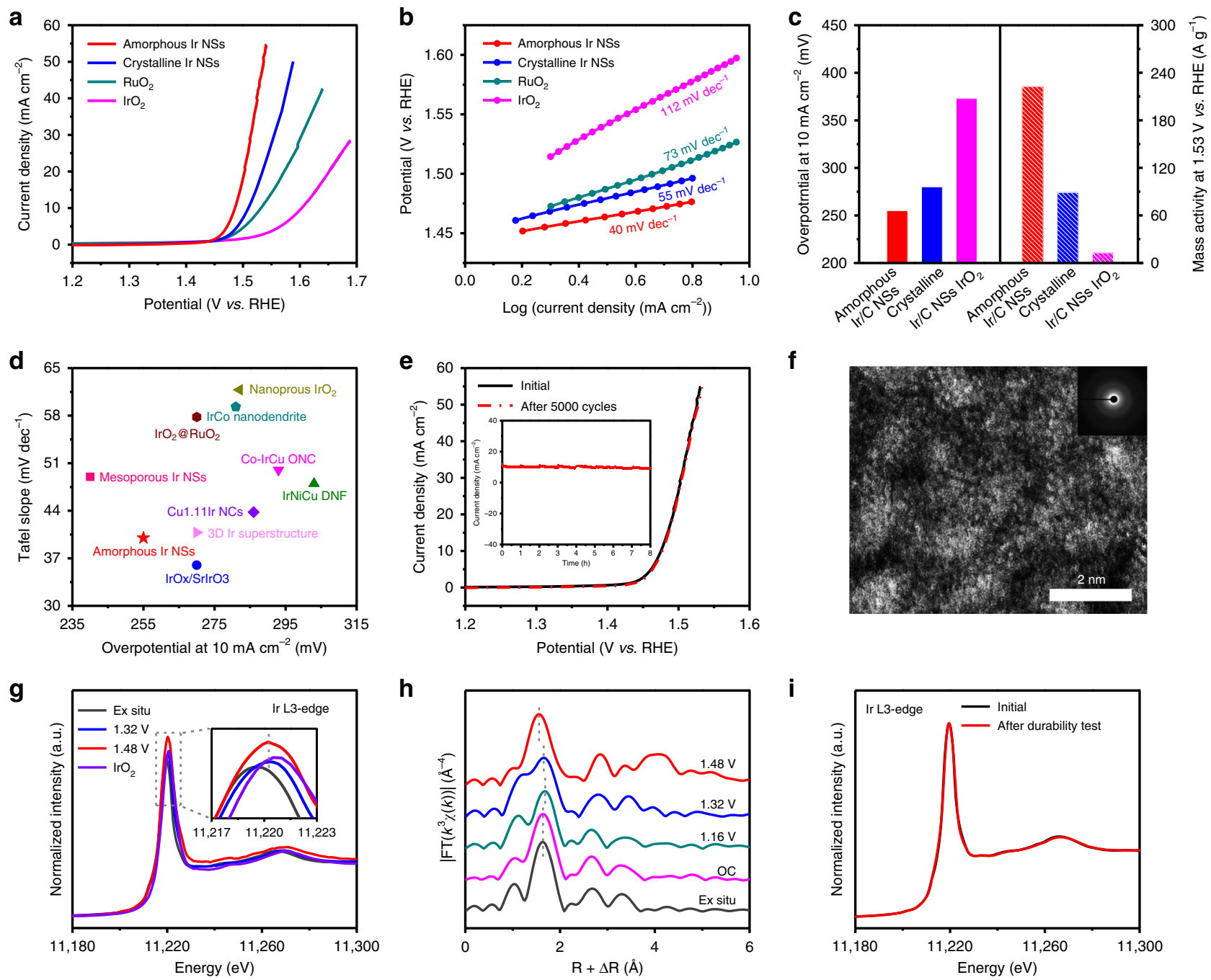

Fig. 4 Electrochemical OER performance and operando X-ray absorption spectroscopy of amorphous Ir NSs. a Polarization curves of amorphous Ir NSs, crystalline Ir NSs, commercial $\mathrm{RuO}_{2}$ and $\mathrm{IrO}_{2}$ catalysts, respectively. b Corresponding Tafel plots of amorphous Ir NSs, crystalline Ir NSs, commercial RuO ${ }_{2}$ and $\mathrm{IrO}_{2}$ catalysts, respectively. c Overpotentials at $10 \mathrm{~mA} \mathrm{~cm}^{-2}$ (left axis) and mass activity at $1.53 \mathrm{~V}$ (vs. RHE) (right axis) of amorphous Ir NSs,

crystalline Ir NSs, and commercial $\mathrm{IrO}_{2}$ catalyst, respectively. $\mathbf{d}$ Comparison with different representative catalysts under acidic media. e Polarization curves of amorphous Ir NSs before and after 5000 cycles. The inset in e shows chronoamperometry measurement of the amorphous Ir NSs at an overpotential of $255 \mathrm{mV}$ for a 8-h test. Note: All the measurements were performed in $\mathrm{O}_{2}$-saturated $0.1 \mathrm{M} \mathrm{HCIO}_{4}$ solution. $\mathbf{f}$ Atomic resolution HAADF-STEM image of the amorphous Ir NSs after durability test. The inset in $\mathbf{f}$ shows the corresponding SAED pattern. $\mathbf{g}, \mathbf{h}$ In situ XAFS spectra change of the Ir $\mathrm{L}_{3}$-edge. $\mathbf{i}$ XANES spectrum of amorphous Ir NSs after durability test

an overpotential of $300 \mathrm{mV}$ (Fig. 4c and Supplementary Fig. 26), which is 2.5 times and 17.6 times larger than that of the crystalline Ir NSs $\left(88.7 \mathrm{~A} \mathrm{~g}^{-1}\right.$ ) and the $\mathrm{IrO}_{2}\left(12.6 \mathrm{~A} \mathrm{~g}^{-1}\right)$, respectively. Moreover, the amorphous Ir NSs exhibit almost the lowest overpotential for a current density of $10 \mathrm{~mA} \mathrm{~cm}^{-2}$ and the smallest Tafel slope compared with previously reported noble metal electrocatalysts (Fig. 4d and Supplementary Table 2). In order to illustrate the superior OER activity of amorphous Ir NSs, electrochemically active surface area (ECSA) and electrochemical impedance spectroscopy (EIS) were tested. The ECSA can be obtained by calculating double-layer capacitance $\left(C_{\mathrm{dl}}\right)^{39,40}$. As shown in Supplementary Figs. 27, 28, the amorphous Ir NSs present a rather high ECSA, revealing that amorphous Ir NSs have abundant OER active sites. Furthermore, the Nyquist plots (Supplementary Fig. 29) show that the amorphous Ir NSs have the lowest charge-transfer resistance among all the tested catalysts, verifying the faster charge transfer process for amorphous Ir NSs. Furthermore, the turnover frequency (TOF) of catalysts were also calculated at different applied potentials ${ }^{41}$ (Supplementary
Fig. 30). Amorphous Ir NSs show a TOF value of $0.16 \mathrm{~s}^{-1}$ at an overpotential of $300 \mathrm{mV}$, which is $3.1,9.9$, and 22.8 times larger than that of the crystalline Ir NSs $\left(0.052 \mathrm{~s}^{-1}\right), \mathrm{RuO}_{2}\left(0.016 \mathrm{~s}^{-1}\right)$, and $\mathrm{IrO}_{2}\left(0.007 \mathrm{~s}^{-1}\right)$, respectively. Besides activity, stability is another important parameter to evaluate the performance of OER electrocatalysts. Surprisingly, the OER activity of amorphous Ir NSs display negligible degradation even after 5000 cycles (Fig. 4e), indicating the excellent stability of amorphous Ir NSs. This observation coincides well with chronoamperometry measurement (inset of Fig. 4e), in which the current density of amorphous Ir NSs at an overpotential of $255 \mathrm{mV}$ sustained $\sim 90 \%$ after a 8 -h test. Furthermore, XRD pattern and aberration-corrected HAADF-STEM analysis after long-term durability test further exhibit ignorable change in the amorphous atomic structure (Fig. 4f and Supplementary Fig. 31).

In situ XAFS studies of amorphous Ir NSs during OER process. To track the evolution process of the atomic structures of Ir 
during OER process, in situ XAFS spectra of amorphous Ir NSs were carried out under different applied potentials ${ }^{42,43}$. As depicted in the in situ XANES spectra (Supplementary Figs. 33, 34 ), the location of white line peak is shifted to the higher energy from ex situ condition to $1.32 \mathrm{~V}$ (vs. reversible hydrogen electrode (RHE)), indicating an increase for the valance state of Ir. Moreover, the location of white line peak remains almost unchanged with the potential increased from 1.32 to $1.48 \mathrm{~V}$ (vs. RHE), while the current density raised from $0.5 \mathrm{~mA}$ to $7.6 \mathrm{~mA} \mathrm{~cm}^{-2}$ (Fig. $4 \mathrm{~g}$ ), which implies an invariability in the oxidation state of Ir during the OER process. Notably, the valance state of Ir has always been maintained lower than +4 under all tested potentials. Furthermore, the local environment evolutions of Ir can be investigated by in situ EXAFS spectrum (Fig. 4h). The distance of the first coordination peak (Ir-C/O) slightly increased from the open-circuit (OC) condition to $1.16 \mathrm{~V}$ (vs. RHE), probably owing to the adsorption of water molecule from the electrolyte. The bond length of Ir-C/O decreased while the applied potential increased from 1.16 to 1.48 $\mathrm{V}$ (vs. RHE), which is possibly attributed to the formation of intermediate species (such as $\mathrm{OOH}^{\star}$ ) or oxygen molecule on the surface $^{44}$. Importantly, the valance state of Ir and the distance of Ir-C/O for amorphous Ir NSs can recover to its initial state after restoring back to the original potential (Supplementary Fig. 35). Moreover, the XAFS spectra of $\mathrm{Ir}_{3}$-edge remains unchanged after a 8-h durability test (Fig. 4i and Supplementary Fig. 36), further revealing the stability of amorphous Ir NSs.

\section{Discussion}

In summary, we demonstrate a general and efficient method to fabricate amorphous noble metal NSs by directly annealing the mixture of metal acetylacetonate and alkali salts. Besides monometal NSs, dozens of different amorphous bimetal NSs and trimetal NSs can be successfully and conveniently prepared. Impressively, the as-prepared amorphous Ir NSs were used as electrocatalyst for OER under acidic media, exhibiting superior electrocatalytic performance compared to crystalline Ir NSs and most reported noble metal electrocatalysts. In situ XAFS spectra reveal that the valance state of Ir has always been maintained lower than +4 during the OER catalysis process. Furthermore, the amorphous atomic structure remains stable after durability test, as demonstrated by aberration-corrected HAADF-STEM image combined with XAFS studies. These findings show that amorphous noble metal materials can not only boost the catalytic activity because of abundant active sites and unique atomic structure but also endow superior catalytic capability that conventional corresponding crystalline catalysts do not afford.

\section{Methods \\ Chemicals. Iridium(III) 2,4-pentanedionate (Ir(acac) $)_{3}$, rhodium(III) 2,4-penta- nedionate $\left(\mathrm{Rh}(\mathrm{acac})_{3}\right)$, iron(III) 2,4-pentanedionate (Fe(acac) 3 ), nickel(II) 2,4- pentanedionate $\left(\mathrm{Ni}(\mathrm{acac})_{2}\right)$, cobalt(III) 2,4-pentanedionate $\left(\mathrm{Co}(\mathrm{acac})_{3}\right)$ were all purchased from Alfa Aesar. Ruthenium acetylacetonate $\left(\mathrm{Ru}(\mathrm{acac})_{3}\right)$ were pur- chased from Adamas-Beta. Sodium nitrate $\left(\mathrm{NaNO}_{3}\right)$, potassium nitrate $\left(\mathrm{KNO}_{3}\right)$, sodium chloride $(\mathrm{NaCl})$, potassium bromide $(\mathrm{KBr})$, and alcohol were all obtained from Sinopharm Chemical Reagent Co., Ltd (Shanghai, China). Ruthenium oxide $\left(\mathrm{RuO}_{2}\right)$ and iridium oxide $\left(\mathrm{IrO}_{2}\right)$ were purchased from Alfa Aesar. Deionized (DI) water from Milli-Q System (Millipore, Billerica, MA) was used in all our experi- ments. Nafion solution ( $5 \mathrm{wt} \%$ ) was purchased from Aldrich. All chemicals were used as received without further purification.}

Synthesis of amorphous Ir NSs. In a typical synthesis of amorphous Ir NSs, 10 $\mathrm{mg} \mathrm{Ir}(\mathrm{acac})_{3}$ and $20 \mathrm{mg} \mathrm{KNO}_{3}$ were dissolved into $7 \mathrm{~mL}$ mixture solution ( $V_{\text {ethanol }} /$ $V_{\text {DI water }}=6 / 1$ ) under magnetic stirring. The mixture solution was dried to obtain the mixed powder. The mixture powder was heated to $300^{\circ} \mathrm{C}$ for $90 \mathrm{~min}$ at the heating rate of $5^{\circ} \mathrm{C} \mathrm{min}-1$ under air in a tube furnace and then naturally cooled to room temperature, yielding the representative samples. The as-obtained products were washed several times with the DI water-ethanol mixing solution.
Synthesis of amorphous Rh NSs, Ru NSs, and RhRu NSs. In a typical synthesis of amorphous $\mathrm{Rh} \mathrm{NSs}, 8 \mathrm{mg} \mathrm{Rh}(\mathrm{acac})_{3}$ and $15 \mathrm{mg} \mathrm{KBr}$ were dissolved into $7 \mathrm{~mL}$ mixture solution $\left(V / V_{\mathrm{DI}}\right.$ water $\left.=6 / 1\right)$ under magnetic stirring. The mixture solution was dried to obtain the mixed powder. The mixture powder was heated to $280-290{ }^{\circ} \mathrm{C}$ for $90 \mathrm{~min}$ under air in a tube furnace and then naturally cooled to room temperature. The as-obtained products were washed several times with the DI water-ethanol mixing solution. The amorphous Ru NSs were obtained using the same process as that of amorphous Rh NSs, except that $8 \mathrm{mg} \mathrm{Rh}(\mathrm{acac})_{3}$ was replaced with $8 \mathrm{mg} \mathrm{Ru}(\mathrm{acac})_{3}$. The amorphous $\mathrm{RhRu}$ NSs were obtained using the same process as that of amorphous Rh NSs, except the addition of $0.4 \mathrm{mg} \mathrm{Ru}$ $(\mathrm{acac})_{3}$.

Synthesis of amorphous IrRu NSs, IrRh NSs, and IrRhRu NSs. In a typical process of amorphous IrRu NSs, $10 \mathrm{mg} \operatorname{Ir}(\mathrm{acac})_{3}, 0.4 \mathrm{mg} \mathrm{Ru}(\mathrm{acac})_{3}$, and $20 \mathrm{mg}$ $\mathrm{KNO}_{3}$ were dissolved into $7 \mathrm{~mL}$ mixture solution $\left(V_{\text {ethanol }} / V_{\text {DI water }}=6 / 1\right)$ under magnetic stirring. The mixture solution was dried to obtain the mixed powder. The mixture powder was heated to $280-290^{\circ} \mathrm{C}$ for $90 \mathrm{~min}$ under air in a tube furnace and then naturally cooled to room temperature. The as-obtained products were washed several times with DI water-ethanol mixture. The amorphous IrRh NSs were obtained using the same process as that of amorphous IrRu NSs, except that $0.4 \mathrm{mg} \mathrm{Ru}(\mathrm{acac})_{3}$ was replaced with $0.4 \mathrm{mg} \mathrm{Rh}(\mathrm{acac})_{3}$. The amorphous IrRhRu NSs were obtained using the same process as that of amorphous IrRu NSs, except the addition of $0.4 \mathrm{mg} \mathrm{Rh}(\mathrm{acac})_{3}$.

Synthesis of amorphous RhFe NSs, RhNi NSs, RhCo NSs, RuFe NSs, RuNi NSs RuCo NSs, IrNi NSs, IrFe NSs, and IrCo NSs. In a typical synthesis of amorphous $\mathrm{RhFe} \mathrm{NSs}, 8 \mathrm{mg} \mathrm{Rh}(\mathrm{acac})_{3}, 0.35 \mathrm{mg} \mathrm{Fe}(\mathrm{acac})_{3}$, and $15 \mathrm{mg} \mathrm{KBr}$ were dissolved into 7 $\mathrm{mL}$ mixture solution $\left(V_{\text {ethanol }} / V_{\text {DI water }}=6 / 1\right)$ under magnetic stirring. The mixture solution was dried to obtain the mixed powder. The mixture powder was heated to $270-280^{\circ} \mathrm{C}$ for $90 \mathrm{~min}$ under air in a tube furnace and then naturally cooled to room temperature. The as-obtained products were washed several times with DI water-ethanol mixture. The amorphous RhNi NSs (RhCo NSs) were obtained using the same process as that of amorphous RhFe NSs, except that $0.35 \mathrm{mg} \mathrm{Fe}$ $(\mathrm{acac})_{3}$ was replaced with $0.25 \mathrm{mg} \mathrm{Ni}(\mathrm{acac})_{2}\left(0.35 \mathrm{mg} \mathrm{Co}(\mathrm{acac})_{3}\right)$. The amorphous RuFe NSs (RuNi NSs, RhCo NSs) were obtained using the same process as that of amorphous RhFe NSs (RhNi NSs, RhCo NSs), except that $8 \mathrm{mg} \mathrm{Rh}(\mathrm{acac})_{3}$ was replaced with $8 \mathrm{mg} \mathrm{Ru}(\mathrm{acac})_{3}$. The amorphous IrFe NSs (IrNi NSs, IrCo NSs) were obtained using the same process as that of amorphous RhFe NSs (RhNi NSs, RhCo $\mathrm{NSs})$, except that $8 \mathrm{mg} \mathrm{Rh}(\mathrm{acac})_{3}$ was replaced with $10 \mathrm{mg} \operatorname{Ir}(\mathrm{acac})_{3}$.

Synthesis of amorphous Ir NSs with a thickness of $\mathbf{3 . 6} \mathbf{~ n m}$ and crystalline Ir NSs. The amorphous Ir NSs with a thickness of $3.6 \mathrm{~nm}$ were obtained using the same process as that of amorphous Ir NSs, except that $20 \mathrm{mg} \mathrm{KNO}_{3}$ was replaced with $18 \mathrm{mg} \mathrm{NaNO}_{3}$. In addition, the crystalline Ir NSs were obtained using the same process as that of amorphous Ir NSs, except that $20 \mathrm{mg} \mathrm{KNO}_{3}$ was replaced with $15 \mathrm{mg} \mathrm{NaCl}(\mathrm{KBr})$.

Characterization. TEM images of all samples were recorded on Hitachi H-7700 operated at $100 \mathrm{kV}$. The SEM and the EDS of samples were carried out by Genimi SEM 500. Aberration-corrected HAADF-STEM images of samples were recorded on JEOL JEM-2010 $\mathrm{LaB}_{6}$ high-resolution TEM and double Cs-corrected JEOL JEM-ARM200CF STEM, which were operated at $200 \mathrm{kV}$. The XRD patterns of samples were conducted on Rigaku Miniflex-600 operating at the voltage of $40 \mathrm{kV}$ and the current of $15 \mathrm{~mA}$ with $\mathrm{Cu}$ Ka radiation $(\lambda=1.5406 \AA)$. XPS was measured at beamline BL10B of National Synchrotron Radiation Laboratory (NSRL) of China using $\mathrm{Mg} \mathrm{Ka}(h v=1248.6 \mathrm{eV})$ radiation source. AFM image was captured by Dimension ICON with Nano Scope V controller (Bruker) in Scan Asyst and tapping mode. The X-ray absorption find structure data ( $\mathrm{Ir} \mathrm{L}_{3}$-edge) was collected at BL14W1 beamline of Shanghai Synchrotron Radiation Facility (SSRF) operated at $3.5 \mathrm{GeV}$ under "top-up" mode with a constant current of $240 \mathrm{~mA}$. The acquired EXAFS data were processed according to the standard procedures using the ATHENA module implemented in the IFEFFIT software packages.

Electrochemical measurements for OER. All the electrochemical experiments were conducted on the CHI 760E electrochemical workstation (Shanghai Chenhua, China) in a conventional three-electrode system at room temperature. Samples were tested on the GCE as the working electrode (WE), $\mathrm{Ag} / \mathrm{AgCl}$ as the reference electrodes, and $\mathrm{Pt}$ wire as the counter electrode (CE). The catalyst solutions were prepared by mixing $2.0 \mathrm{mg}$ catalysts with $2.0 \mathrm{mg}$ carbon black (Cabot Vulcan XC72) in a solution containing $490 \mu \mathrm{L}$ of ethanol, $490 \mu \mathrm{L}$ of $\mathrm{H}_{2} \mathrm{O}$, and $20 \mu \mathrm{L}$ of $5 \mathrm{wt} \%$ Nafion solution by sonication to form homogeneous catalyst inks. Then, $20 \mu \mathrm{L}$ well-dispersed catalyst ink was carefully dropped onto the polished glassy carbon rotating disk electrode with drying naturally for testing.

OER tests were conducted in $\mathrm{O}_{2}$-saturated $0.1 \mathrm{M} \mathrm{HClO}_{4}$ solution with a scan rate of $5 \mathrm{mV} \mathrm{s}^{-1}$ at 1600 r.p.m. on a rotating electrode. All potentials were referenced to an RHE with IR correction, where the $\mathrm{R}$ was referred to the ohmic resistance arising from the electrolyte/contact resistance of the setup and measured by EIS. EIS measurements were carried out from $100 \mathrm{kHz}$ to $0.1 \mathrm{~Hz}$ on a rotation electrode under 1600 r.p.m. 
ECSA calculation. The ECSA was estimated from the electrochemical double-layer capacitance $\left(C_{\mathrm{dl}}\right)$ of the catalytic surface. The $C_{\mathrm{dl}}$ was determined by measuring the non-Faradaic capacitive current charging from the scan rates dependence of cyclic voltammograms. The double layer capacitance $\left(C_{\mathrm{dl}}\right)$ is obtained from the charge current $\left(i_{c}\right)$ as function of the scan rate $(v)$, which is equal to the slope based on Eq. (1) shown as follows:

$$
C_{\mathrm{dl}}=\frac{i_{\mathrm{c}}}{v}
$$

ECSA is calculated using Eq. (2), whereas the specific capacitance $\left(C_{\mathrm{s}}\right)$ is 0.035 $\mathrm{mF} \mathrm{cm}-2$ in $0.1 \mathrm{M} \mathrm{HClO}_{4}$ aqueous solution:

$$
\mathrm{ECSA}=\frac{C_{\mathrm{dl}}}{C_{\mathrm{S}} \times m_{\text {catalyst }}} .
$$

TOF calculation. The TOF was calculated based on the method reported in previous works. This calculation assumes 100\% Faradaic efficiency:

$$
\mathrm{TOF}=\frac{N_{\mathrm{O}_{2}}}{N_{\text {metal }}},
$$

where $N_{\mathrm{O}_{2}}$ is the number of $\mathrm{O}_{2}$ turnovers, calculated using the following formula:

$$
N_{\mathrm{O}_{2}}=\frac{j\left(\frac{A}{\mathrm{~cm}^{2}}\right) \times \mathrm{Scm}_{\text {oxide }}^{2} \times 1 \frac{\mathrm{C}}{\mathrm{s}} \times 1 \mathrm{~mol} \mathrm{e}^{-}}{96,485 \mathrm{C} \times 4 e^{-}} \times N_{\mathrm{A}},
$$

where $j$ is the measured current density, $A$ is the surface area of electrode, and $N_{\mathrm{A}}$ is Avogadro constant $\left(6.02 \times 10^{23} \mathrm{~mol}^{-1}\right)$.

The number of metal sites $\left(N_{\text {metal }}\right)$ is only calculated the metal (Ir or Ru) number sites in tested catalyst.

In situ XAFS measurements. Electrochemical measurements were conducted on a computer-controlled electrochemical analyzer (Supplementary Fig. 32). Catalystmodified carbon paper was used as the WE, Pt wire as the counter electrode (CE) and $\mathrm{Ag} / \mathrm{AgCl}$ electrode as the reference electrode (RE). In situ XAFS was used to probe the valence state and coordination environment for amorphous Ir NSs during OER process. The dilute catalyst slurry $(50 \mu \mathrm{l}$ ink) was evently distributed on the carbon paper $(3 \mathrm{~cm} \times 3 \mathrm{~cm})$.

Collection and analysis of RDF. The microstructure and SAED of the samples were investigated by double Cs-corrected JEOL JEM-ARM200CF scanning TEM that was operated at $200 \mathrm{kV}$. The conversion of SAED pattern (Fig. $2 \mathrm{c}$ and Supplementary Fig. 22) into the radial distribution function $g(r)$ was conducted using the freely available PASAD-tools package for the GATAN Digital micrograph software.

\section{Data availability}

The data that support the findings of this study are available from the corresponding authors upon reasonable request.

Received: 24 July 2019; Accepted: 30 September 2019; Published online: 24 October 2019

\section{References}

1. Corma, A. et al. Exceptional oxidation activity with size-controlled supported gold clusters of low atomicity. Nat. Chem. 5, 775-781 (2013).

2. Yao, Y. et al. Carbothermal shock synthesis of high-entropy-alloy nanoparticles. Science 359, 1489-1494 (2018).

3. Ding, K. et al. A general synthesis approach for supported bimetallic nanoparticles via surface inorganometallic chemistry. Science 362, 560-564 (2018).

4. Hunt, S. T. et al. Self-assembly of noble metal monolayers on transition metal carbide nanoparticle catalysts. Science 352, 974-978 (2016).

5. Zhai, Y. et al. Alkali-stabilized Pt-OHx species catalyze low-temperature water-gas shift reactions. Science 329, 1633-1636 (2010).

6. Strasser, P. et al. Lattice-strain control of the activity in dealloyed core-shell fuel cell catalysts. Nat. Chem. 2, 454 (2010).

7. Seh, Z. W. et al. Combining theory and experiment in electrocatalysis: insights into materials design. Science 355, eaad4998 (2017).

8. Spoeri, C., Kwan, J. T. H., Bonakdarpour, A., Wilkinson, D. P. \& Strasser, P. The stability challenges of oxygen evolving catalysts: Towards a common fundamental understanding and mitigation of catalyst degradation. Angew. Chem. Int. Ed. 56, 5994-6021 (2017).

9. Reier, T., Nong, H. N., Teschner, D., Schlögl, R. \& Strasser, P. Electrocatalytic oxygen evolution reaction in acidic environments-reaction mechanisms and catalysts. Adv. Energy Mater. 7, 1601275 (2017).
10. Xia, Y., Xiong, Y., Lim, B. \& Skrabalak, S. E. Shape-controlled synthesis of metal nanocrystals: simple chemistry meets complex physics? Angew. Chem. Int. Ed. 48, 60-103 (2009).

11. Zhou, J. et al. A library of atomically thin metal chalcogenides. Nature 556, 355-359 (2018).

12. Yang, N. et al. Synthesis of ultrathin PdCu alloy nanosheets used as a highly efficient electrocatalyst for formic acid oxidation. Adv. Mater. 29, 1700769 (2017).

13. Fan, Z. \& Zhang, H. Crystal phase-controlled synthesis, properties and applications of noble metal nanomaterials. Chem. Soc. Rev. 45, 63-82 (2016).

14. Lu, Q. et al. Crystal phase-based epitaxial growth of hybrid noble metal nanostructures on $4 \mathrm{H} / \mathrm{fcc}$ Au nanowires. Nat. Chem. 10, 456-461 (2018).

15. Huang, X. et al. Synthesis of hexagonal close-packed gold nanostructures. Nat. Commun. 2, 292 (2011).

16. Tsuji, E., Imanishi, A., Fukui, K.-i \& Nakato, Y. Electrocatalytic activity of amorphous $\mathrm{RuO} 2$ electrode for oxygen evolution in an aqueous solution. Electrochim. Acta 56, 2009-2016 (2011).

17. Li, M. et al. Ultrafine jagged platinum nanowires enable ultrahigh mass activity for the oxygen reduction reaction. Science 354, 1414-1419 (2016).

18. Wang, L. et al. Tunable intrinsic strain in two-dimensional transition metal electrocatalysts. Science 363, 870-874 (2019).

19. Zhang, Z. et al. Submonolayered Ru deposited on ultrathin Pd nanosheets used for enhanced catalytic applications. Adv. Mater. 28, 10282-10286 (2016)

20. Hirata, A. et al. Direct observation of local atomic order in a metallic glass. Nat. Mater. 10, 28-33 (2011).

21. Zeng, Q. et al. Long-range topological order in metallic glass. Science 332, 1404-1406 (2011).

22. Wagner, H. et al. Local elastic properties of a metallic glass. Nat. Mater. 10, 439-442 (2011).

23. Zhang, Y., Wang, W. \& Greer, A. Making metallic glasses plastic by control of residual stress. Nat. Mater. 5, 857-860 (2006).

24. Hofmann, D. C. et al. Designing metallic glass matrix composites with high toughness and tensile ductility. Nature 451, 1085-1089 (2008).

25. Pei, Y. et al. Synthesis and catalysis of chemically reduced metal-metalloid amorphous alloys. Chem. Soc. Rev. 41, 8140-8162 (2012).

26. Jia, B. et al. Creating ultrathin amorphous metal hydroxide and oxide nanosheets libraries. J. Mater. Chem. A 7, 4383-4388 (2019).

27. Smith, R. D. et al. Photochemical route for accessing amorphous metal oxide materials for water oxidation catalysis. Science 340, 60-62 (2013).

28. Cheng, H. et al. Aging amorphous/crystalline heterophase PdCu nanosheets for catalytic reactions. Natl Sci. Rev. https://doi.org/10.1093/nsr/nwz078 (2019).

29. Zhang, B. et al. Homogeneously dispersed multimetal oxygen-evolving catalysts. Science 352, 333-337 (2016)

30. Zhang, X. et al. Lithiation-induced amorphization of Pd 3 P 2 S 8 for highly efficient hydrogen evolution. Nat. Catal. 1, 460-468 (2018).

31. Yang, N. et al. Amorphous/crystalline hetero-phase Pd nanosheets: one-pot synthesis and highly selective hydrogenation reaction. Adv. Mater. 30, 1803234 (2018)

32. Cheng, H., Yang, N., Lu, Q., Zhang, Z. \& Zhang, H. Syntheses and properties of metal nanomaterials with novel crystal phases. Adv. Mater. https://doi.org/ 10.1002/adma.201707189 (2018).

33. Shan, C.-C., Tsai, D.-S., Huang, Y.-S., Jian, S.-H. \& Cheng, C.-L. Pt-Ir - IrO2NT Thin-wall electrocatalysts derived from IrO2 nanotubes and their catalytic activities in methanol oxidation. Chem. Mater. 19, 424-431 (2007).

34. Slavcheva, E., Borisov, G., Lefterova, E., Petkucheva, E. \& Boshnakova, I. Ebonex supported iridium as anode catalyst for PEM water electrolysis. Int. J. Hydrog. Energy 40, 11356-11361 (2015).

35. Gammer, C., Mangler, C., Rentenberger, C. \& Karnthaler, H. Quantitative local profile analysis of nanomaterials by electron diffraction. Scr. Mater. 63, 312-315 (2010).

36. Wei, S. et al. X-ray-absorption fine structure study on devitrification of ultrafine amorphous Ni-B alloys. Phys. Rev. B 63, 224201 (2001).

37. Debenedetti, P. G. \& Stillinger, F. H. Supercooled liquids and the glass transition. Nature 410, 259-267 (2001)

38. Swallen, S. F. et al. Organic glasses with exceptional thermodynamic and kinetic stability. Science 315, 353-356 (2007).

39. McCrory, C. C. et al. Benchmarking hydrogen evolving reaction and oxygen evolving reaction electrocatalysts for solar water splitting devices. J. Am. Chem. Soc. 137, 4347-4357 (2015).

40. Shi, Q. et al. Nanovoid incorporated Ir $\mathrm{x} \mathrm{Cu}$ metallic aerogels for oxygen evolution reaction catalysis. ACS Energy Lett. 3, 2038-2044 (2018).

41. Lin, Y. et al. Chromium-ruthenium oxide solid solution electrocatalyst for highly efficient oxygen evolution reaction in acidic media. Nat. Commun. 10, 162 (2019).

42. Gao, J. et al. Breaking long-range order in iridium oxide by alkali ion for efficient water oxidation. J. Am. Chem. Soc. 141, 3014-3023 (2019). 
43. Yao, Y. et al. Engineering the electronic structure of single atom Ru sites via compressive strain boosts acidic water oxidation electrocatalysis. Nat. Catal. 2, 304-313 (2019).

44. Cao, L. et al. Identification of single-atom active sites in carbon-based cobalt catalysts during electrocatalytic hydrogen evolution. Nat. Catal. 2, 134-141 (2019).

\section{Acknowledgements}

This work was supported by the National Key R\&D Program of China (2017YFA0700104), National Natural Science Foundation of China (21571169, 21871238, 11875258, 11505187, and 41701359), Fundamental Research Funds for the Central Universities (WK2060190081), Youth Innovation Promotion Association of the Chinese Academy of Science (2018494), and Young Elite Scientists Sponsorship Program by CAST (2016QNRC001). We thank the photoemission end stations BL10B in NSRL and BL14W1 in SSRF for help in characterizations.

\section{Author contributions}

Y.L. and X.H. conceived the idea and co-wrote the paper. G.W. carried out the sample synthesis, characterization, and OER measurement. X.Z., P.C., and W.C. performed the XAFS characterization. Y.L. and Q.Z. performed the aberration-corrected HAADFSTEM characterizations. H.J. and L.G. calculated the RDF characterization. H.L. carried out the AFM characterization. X.W. and Y.Q. helped with the modification of the paper. X.H., Y.H., P.L., J.G., Y.Y., R.S., and Y.W. discussed the results and commented on the manuscript.

\section{Competing interests}

The authors declare no competing interests.

\section{Additional information}

Supplementary information is available for this paper at https://doi.org/10.1038/s41467019-12859-2.

Correspondence and requests for materials should be addressed to X.H. or Y.L.

Peer review information Nature Communications thanks anonymous reviewers for their contributions to the peer review of this work.

Reprints and permission information is available at http://www.nature.com/reprints

Publisher's note Springer Nature remains neutral with regard to jurisdictional claims in published maps and institutional affiliations.

(c) (i) Open Access This article is licensed under a Creative Commons Attribution 4.0 International License, which permits use, sharing, adaptation, distribution and reproduction in any medium or format, as long as you give appropriate credit to the original author(s) and the source, provide a link to the Creative Commons license, and indicate if changes were made. The images or other third party material in this article are included in the article's Creative Commons license, unless indicated otherwise in a credit line to the material. If material is not included in the article's Creative Commons license and your intended use is not permitted by statutory regulation or exceeds the permitted use, you will need to obtain permission directly from the copyright holder. To view a copy of this license, visit http://creativecommons.org/ licenses/by/4.0/.

(C) The Author(s) 2019 